\title{
QFD PARA ENGENHARIA SIMULTÂNEA BASEADA EM CONJUNTOS (SBCE): O DESENVOLVIMENTO DE UM REFRIGERADOR MAGNÉTICO COMPACTO
}

Mayara Silvestre de Oliveira (mayarasilvestredeoliveira@gmail.com) - Departamento de Engenharia Mecânica, Universidade Federal de Santa Catarina.

Jaime Andrés Lozano Cadena (jaime@polo.ufsc.br) - Departamento de Engenharia Mecânica, Universidade Federal de Santa Catarina.

Jader Riso Barbosa Jr. (jrb@polo.ufsc.br) - Departamento de Engenharia Mecânica, Universidade Federal de Santa Catarina.

Fernando Antônio Forcellini (forcellini@gmail.com) - Departamento de Engenharia Mecânica, Universidade Federal de Santa Catarina.

\section{RESUMO}

A Engenharia Simultânea baseada em Conjuntos, conhecida em inglês como Set-Based Concurrent Engineering (SBCE), fundamenta-se no afunilamento do espaço de projeto, que ocorre pela intersecção das alternativas de solução entre os subsistemas. Para isso, existe a necessidade de desdobrar os requisitos do cliente para cada subsistema e de estudar os trade-offs de projeto para que se possa afunilar as soluções gradativamente. Entretanto, foi percebida na literatura uma ausência em como desdobrar os requisitos do cliente para os subsistemas e como definir os trade-offs de projeto em um ambiente de SBCE. Dentro desse contexto, este trabalho tem por objetivo demonstrar o uso do QFD em um ambiente de SBCE para desdobrar o valor do produto para seus correspondentes subsistemas. Além disso, demonstra-se como o QFD pode fornecer um panorama dos trade-offs de projeto dentro de cada subsistema e entre eles. Neste trabalho foi realizada a aplicação do QFD nos estágios iniciais do desenvolvimento de um refrigerador magnético compacto em um ambiente de SBCE. Através da aplicação do QFD, obteve-se um desdobramento dos requisitos do cliente e do valor do produto para os subsistemas. Além disso, construiu-se um panorama dos trade-offs de projeto através das correlações obtidas nas matrizes do QFD. Observou-se um alinhamento da equipe em relação ao valor, o que permitiu a integração dos subsistemas durante o desenvolvimento. Foi possível utilizar o QFD como uma primeira restrição do espaço de projeto, à medida que se definiram as faixas de valores como metas para cada característica de engenharia na casa da qualidade do sistema e em cada matriz de características dos subsistemas. Os trade-offs de projeto encontrados serviram de insumo para o afunilamento do espaço de projeto. Cada trade-off de projeto foi estudado e suas respectivas curvas foram geradas dentro de cada subsistema e entre eles. Isso permitiu a delimitação do espaço de projeto, a comparação desse espaço entre os subsistemas buscando as intersecções e consequentemente, descartando as regiões do espaço de projeto que não se mostraram viáveis ou compatíveis com os demais subsistemas.

Palavras-chave: Quality function deployment; desenvolvimento de produtos lean; engenharia simultânea baseada em conjuntos; trade-off; refrigeração magnética

Área: Gestão do Processo de Desenvolvimento de Produtos

\section{INTRODUÇÃO}


A engenharia simultânea baseada em conjuntos (SBCE) é a estratégia de convergência de conceitos utilizada no desenvolvimento lean de produtos. A SBCE que preconiza o afunilamento gradativo das possíveis alternativas de projeto, através da eliminação das soluções que se mostram menos adequadas que as demais. Esse afunilamento se faz possível através do desdobramento dos requisitos do cliente para os subsistemas e da definição dos trade-offs de projeto. Os trade-offs de projeto possuem papel chave nesse afunilamento. (SOBEK II; WARD; LIKER, 1999, RAUDBERGET, 2010, MCKENNEY; KEMINK; SINGER, 2011, WARD, 2011, ARACI; AL-ASHAAB; MAKSIMOVIC, 2016). Entretanto, uma ausência na literatura foi percebida em relação a como encontrar estes trade-offs. Araci et al. (2016) desenvolveram um modelo para realizar os estudos dos trade-offs, entretanto, não explicitam como encontrá-los.

Uma ferramenta consolidada para o desdobramento dos requisitos do cliente é o Quality Function Deployment (QFD), entretanto, não se esclarece na literatura, como ela pode ser utilizada em um ambiente de SBCE e, também, como se pode utilizá-la para obter um panorama dos trade-offs de projeto. Portanto, o presente artigo tem como objetivo demonstrar como o QFD pode ser utilizado em um ambiente de SBCE para realizar o desdobramento do valor para os subsistemas e para encontrar os trade-offs de projeto. Para tanto, inicialmente apresenta-se o uso da ferramenta no SBCE e sua aplicação no desenvolvimento de um refrigerador magnético compacto. Por fim, os resultados da aplicação são analisados.

\section{METODOLOGIA}

A metodologia utilizada para este trabalho foi uma pesquisa ação, conforme é apresentada na Figura 1.

Figura 1. Metodologia utilizada.

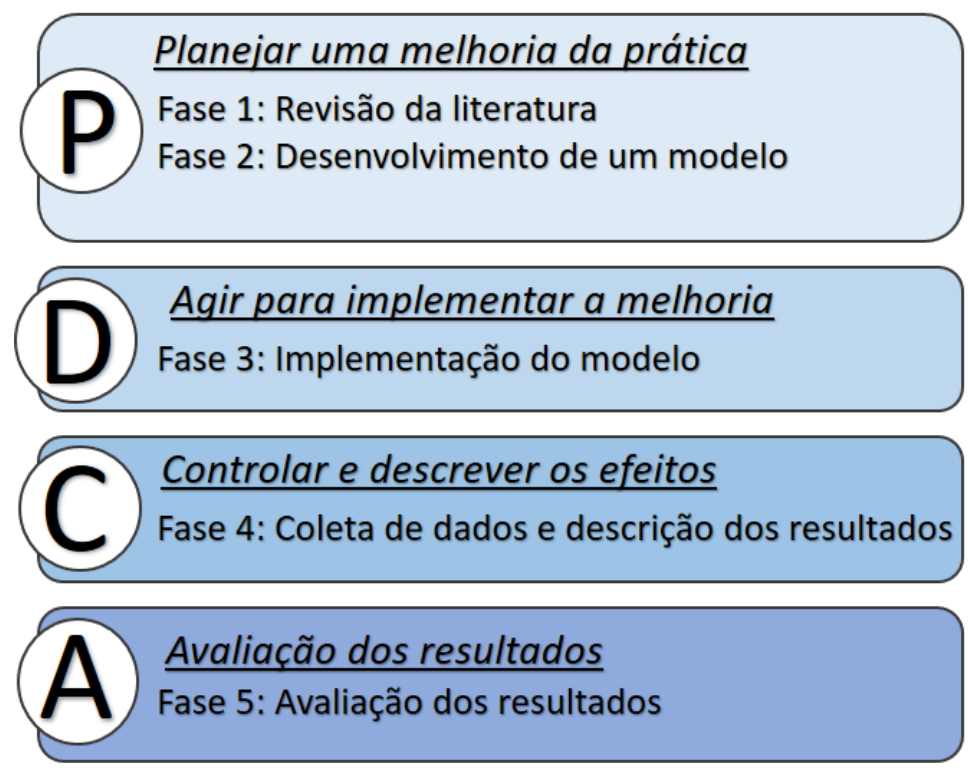

Inicialmente, na primeira fase, uma revisão da literatura foi realizada para encontrar as abordagens existentes acerca de desdobramento do valor e SBCE. Com base nos resultados da primeira fase, um modelo sobre como desdobrar os requisitos do cliente para os subsistemas do produto e encontrar os trade-offs de projeto foi elaborado. Na terceira fase, foi realizada uma aplicação do modelo nos estágios iniciais do desenvolvimento de um produto para 
demostrar os resultados provenientes do seu uso para o SBCE. Durante a aplicação do modelo, foram coletados dados e os resultados foram descritos. Por fim, uma análise dos resultados obtidos foi feita.

\section{REFERENCIAL TEÓRICO}

A SBCE é uma estratégia de convergência de conceitos gerados durante o desenvolvimento de produtos. Essa estratégia se fundamenta em desenvolver conjuntos de soluções em paralelo e as afunilar gradativamente, de forma a eliminar as alternativas que se mostram menos viáveis. Esse afunilamento ocorre através da comparação das possíveis soluções do projeto com os requisitos do cliente e com as demais soluções e também através do estudo dos tradeoffs de projeto. Aquelas alternativas que se mostram incompatíveis com as demais soluções ou ainda, mostram que não irão atendem aos requisitos do cliente são eliminadas ao longo do desenvolvimento. Ao final do processo, apenas uma solução irá restar, que será a solução adotada para o produto (FORD; SOBEK II, 2005).

A SBCE explora os trade-offs para afunilar as alternativas para o projeto até que a solução final seja obtida (WARD, 2011). Durante a etapa do projeto conceitual, existem vários parâmetros conflitantes entre si, o que caracteriza os trade-offs de projeto. Esses parâmetros são bastante impactantes na tomada de decisão do projeto. Portanto, é importante identificar esses parâmetros conflitantes e entender a relação entre eles de forma visual. Esse é um importante passo na SBCE (ARACI, AL-ASHAAB, MAKSIMOVIC, 2016), a qual se inicia dividindo o produto em subsistemas. Cada subsistema irá estudar e desenvolver conjuntos de soluções e terá suas próprias restrições advindas das restrições gerais do produto (WARD, 2011). Para que o subsistema consiga afunilar suas alternativas de projeto é preciso que ocorra o chamado desdobramento do valor. Ele serve para que restrições sejam impostas para assegurar que a solução final de um subsistema, escolhida de um conjunto de alternativas, irá trabalhar em concordância com todas as soluções finais dos outros subsistemas (BAINES et al., 2006, SINGER; DOERRY; BUCKLEY, 2009). Consequentemente, a importância do desdobramento do valor se dá pela compatibilidade entre todos os subsistemas para formar um produto final que integre todas essas soluções.

Em um ambiente de SBCE, o desdobramento do valor é feito por matrizes que comparam e qualificam conceitos que são utilizadas para auxiliar a tomada de decisão (KHAN et al., 2013). Essas matrizes são oriundas do QFD, uma ferramenta de planejamento cujo objetivo é estudar a demanda, coletar e identificar as necessidades dos consumidores e definir os seus requisitos de forma sistemática (DIKMEN, BIRGONUL, KIZILTAS, 2005). Nos primeiros estágios do desenvolvimento de produtos, as necessidades dos clientes registradas no QFD são transformadas em requisitos para o projeto e utilizadas ao longo do processo de desenvolvimento como suporte para a tomada de decisão (NATEE; LOW; TEO, 2016). O QFD tem a função de apoiar a pesquisa e o desenvolvimento do produto através da especificação das características do produto, processos e materiais para que os requisitos do cliente sejam alcançados (CHENG, 2002). No QFD, inicialmente lista-se os requisitos do consumidor e durante o desenvolvimento, esses requisitos são convertidos em especificações do projeto. Essas especificações do projeto são traduzidas então em especificações técnicas detalhadas para os subsistemas (FRANCESCHINI, 2001).

O QFD consiste em dois elementos: matrizes bidimensionais e um framework que conecte essas matrizes (MARITAN, 2015). As matrizes consistem em quatro tipos: matriz de planejamento do produto, matriz de desdobramento em subsistemas, matriz de planejamento do processo e matriz de controle do processo. Elas estão distribuídas em diferentes níveis, nos 
quais as linhas representam os requisitos do cliente e as colunas as características de engenharia do produto, conforme apresentado na Figura 2 (FRANCESCHINI, 2001).

Figura 2. Desdobramento do valor no QFD. Fonte: Adaptado de Franceschini (2001)

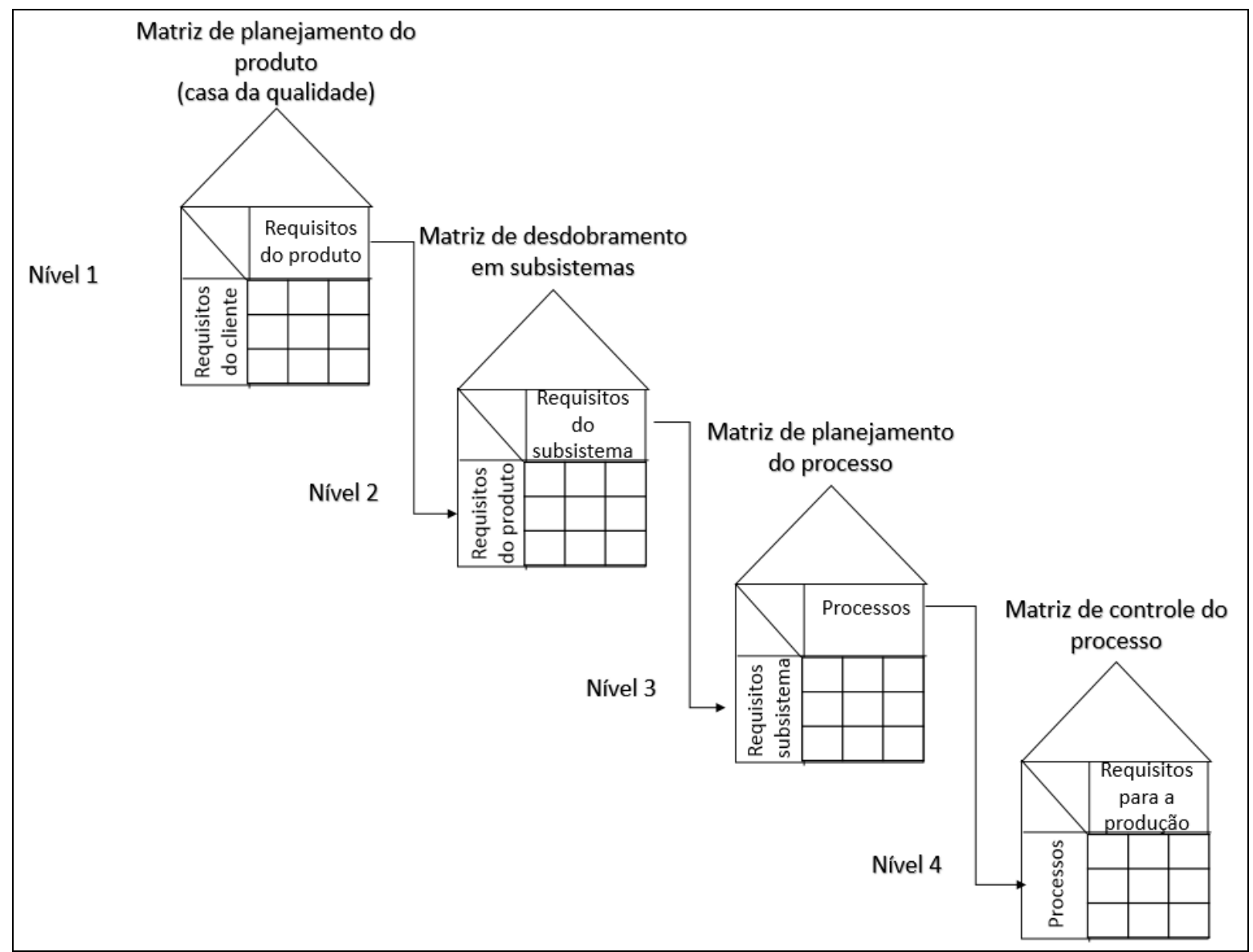

As matrizes permitem um enfoque em aspectos chave de várias funções em níveis de detalhe variáveis (FRANCESCHINI, 2001). O desdobramento do QFD segue com o output de cada matriz servindo como input para a próxima, ou seja, as colunas da matriz anterior serão as linhas da matriz posterior (MARITAN, 2015). O primeiro nível do QFD é a chamada casa da qualidade, cujo foco é o produto. Ela correlaciona as necessidades do cliente e as características do produto (NATEE; LOW; TEO, 2016). Para a construção da matriz do primeiro nível, lista-se inicialmente os requisitos do consumidor, definidos através de um estudo das necessidades dos clientes. Esses requisitos são então, priorizados à luz de uma avaliação da sua importância para o cliente. A seguir, as características técnicas que definem o produto são listadas e a priorização e a correlação entre essas características são analisadas. Por fim, uma correlação entre os requisitos e as características é feita.

O segundo nível do QFD corresponde ao desdobramento do valor do produto para suas partes e compara as características do produto com os requisitos dos componentes ou subsistemas. $\mathrm{O}$ terceiro nível do QFD relaciona as características dos subsistemas com seu processo de produção, em que se identifica parâmetros críticos do produto e os detalha para adequar os processos de fabricação. Por fim, o quarto nível define parâmetros e métodos relacionados a inspeção e controle da qualidade no processo de produção. Dessa forma, são detalhados parâmetros de controle do processo, pontos e métodos de controle, tamanho da amostra e frequência de conferência (FRANCESCHINI, 2001). 


\section{QFD PARA A ENGENHARIA SIMULTÂNEA BASEADA EM CONJUNTOS}

Uma das funções do QFD na SBCE deve ser a de desdobrar o valor esperado do produto para cada subsistema. Ao final do desenvolvimento, para formar o produto, os subsistemas devem ser integráveis e compatíveis. Quando não existe clareza sobre o papel e o desempenho de cada subsistema para esse produto, os riscos associados aos problemas de integração dos subsistemas aumentam. Portanto, as matrizes do QFD e o seu framework promovem clareza, integração e alinhamento dos subsistemas. O desdobramento do valor entre os subsistemas ocorre conforme é apresentado na Figura 3.

Figura 3. Desdobramento do valor entre os subsistemas.

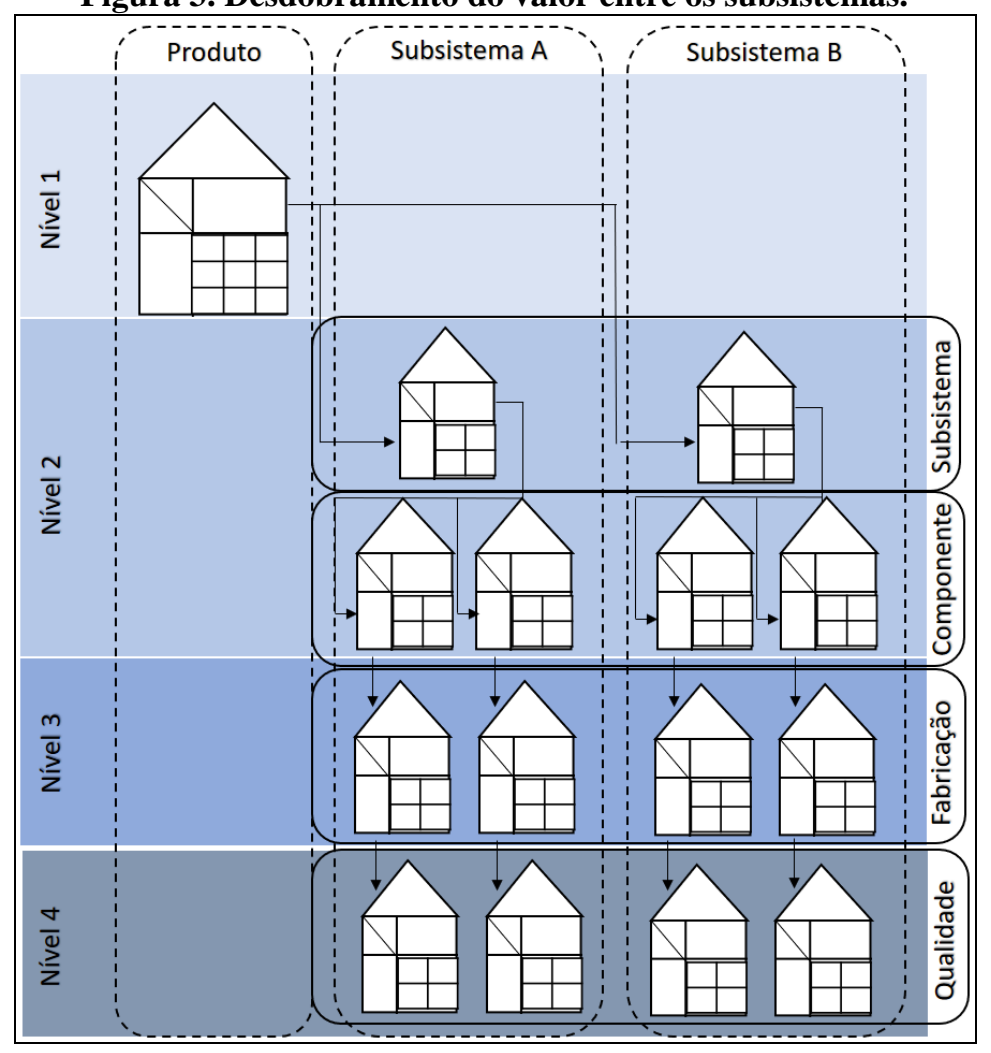

Inicialmente, a matriz da casa da qualidade deve ser construída através de estudos sobre os requisitos do cliente e suas expectativas quanto ao produto. Essa matriz se desdobra em várias matrizes para cada subsistema associado ao produto. A conexão entre a casa da qualidade e o segundo nível se dá pelas características de engenharia do produto, que se torna os requisitos do cliente para os subsistemas. Quando se conecta ao subsistema as características de engenharia que o produto deve ter, cria-se clareza sobre qual é o papel que o subsistema tem na entrega do valor e quais as metas ele deve alcançar para que o produto possua $o$ funcionamento esperado pelo cliente. Ainda no nível do subsistema, podem haver componentes considerados críticos para o projeto. Nesse caso, pode-se escolher desdobrar mais uma vez as matrizes para o nível de peças e componentes. Dessa forma, as características de engenharia do subsistema serão os requisitos do cliente para o componente. Conhece-se então qual o desempenho necessário que esse componente deve ter para que o subsistema entregue o valor esperado.

O próximo desdobramento ocorre para o terceiro nível, o processo de manufatura do componente. Nesse nível, as características de engenharia do componente serão os requisitos 
para o processo de produção. Especificações e exigências de materiais, desempenho, tolerâncias de fabricação são explicitadas nesse nível. A partir daí é possível desdobrar para o último nível, que é a qualidade necessária ao processo de fabricação. As características necessárias para o desempenho do processo de fabricação serão os requisitos do cliente para o controle da qualidade.

O QFD então forma um framework que desdobra o valor para todos os níveis necessários ao desenvolvimento do produto, criando um entendimento claro e uniforme sobre o valor a todos os desenvolvedores em todos os níveis do projeto. Esse entendimento diminui os riscos associados ao desenvolvimento e torna possível a integração dos subsistemas para afunilar as alternativas de projeto, o que é preconizado pela SBCE. Além disso, as matrizes do QFD devem ser documentos vivos no processo de desenvolvimento para que a medida que mais conhecimento sobre o produto é adquirido, mais refinadas as matrizes ficam. $\mathrm{O}$ espaço de projeto do produto e dos subsistemas deve estar explicitado nas matrizes, conforme mostra a Figura 4.

Figura 4. QFD e o afunilamento do espaço de projeto na SBCE.

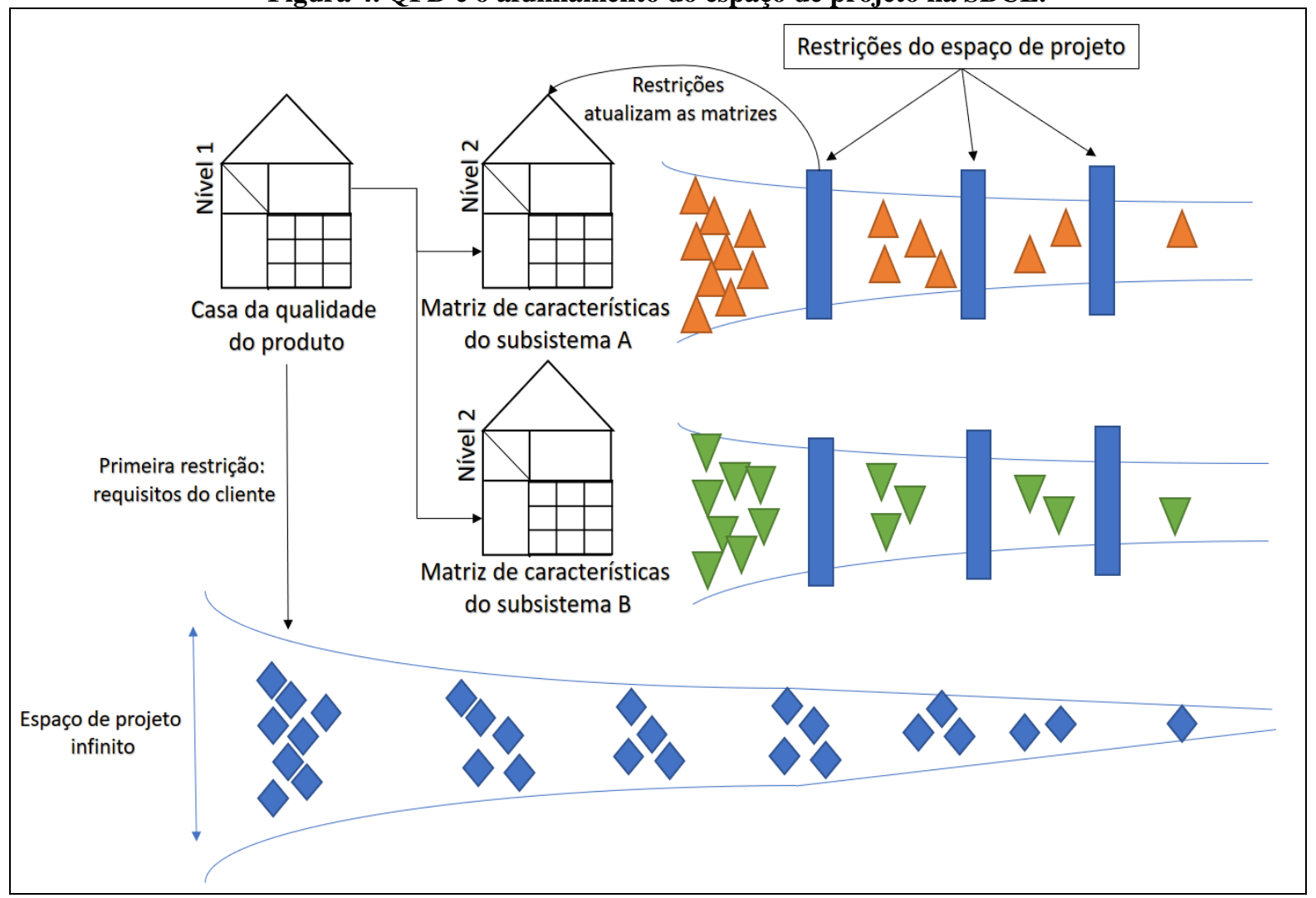

Inicialmente, antes de construir a casa da qualidade do produto, as características de engenharia podem assumir qualquer valor. Quando restritas à luz dos requisitos do cliente, essas características devem atingir determinadas exigências, através de faixas de valores aceitáveis pelo consumidor. A medida que o desenvolvimento e estudo das alternativas para o projeto acontece, os desenvolvedores percebem que mais restrições podem ser feitas. Portanto, à medida que o tempo de desenvolvimento aumenta, essas faixas vão diminuindo e por consequência o espaço de projeto também diminui. Ao final do processo, restará apenas um ponto no espaço que será o valor adotado para o projeto final do produto. A combinação 
dos pontos de cada subsistema irá ser o produto final. Por essa razão, a integração e compatibilidade entre as soluções dos subsistemas é um dos critérios do afunilamento.

Os trade-offs de projeto podem ser identificados nas matrizes do QFD. Essas matrizes permitem visualizar a correlação entre as especificações técnicas do produto ou de seus subsistemas e as tendências de projeto desejáveis. $O$ trade-off ocorre quando duas características possuem a relação de correlação em discordância com a tendência desejada para o projeto. Em outras palavras, quando duas características são inversamente proporcionais (sinal negativo no teto da matriz do QFD), e a tendência do projeto é aumentar ou diminuí-las simultaneamente, teremos um trade-off. Isso ocorre pois se deseja aumentar ou diminuir as duas variáveis simultaneamente e isso não é possível, pois se aumentarmos uma, a outra consequentemente, irá diminuir. $\mathrm{O}$ mesmo pode ser observado quando desejamos aumentar uma característica e diminuir a outra, quando elas são diretamente proporcionais (sinal positivo no teto da matriz do QFD). Essas situações constituem os trade-offs de projeto, conforme apresentado na Figura 5.

Figura 5. Trade-offs no teto da matriz do QFD.

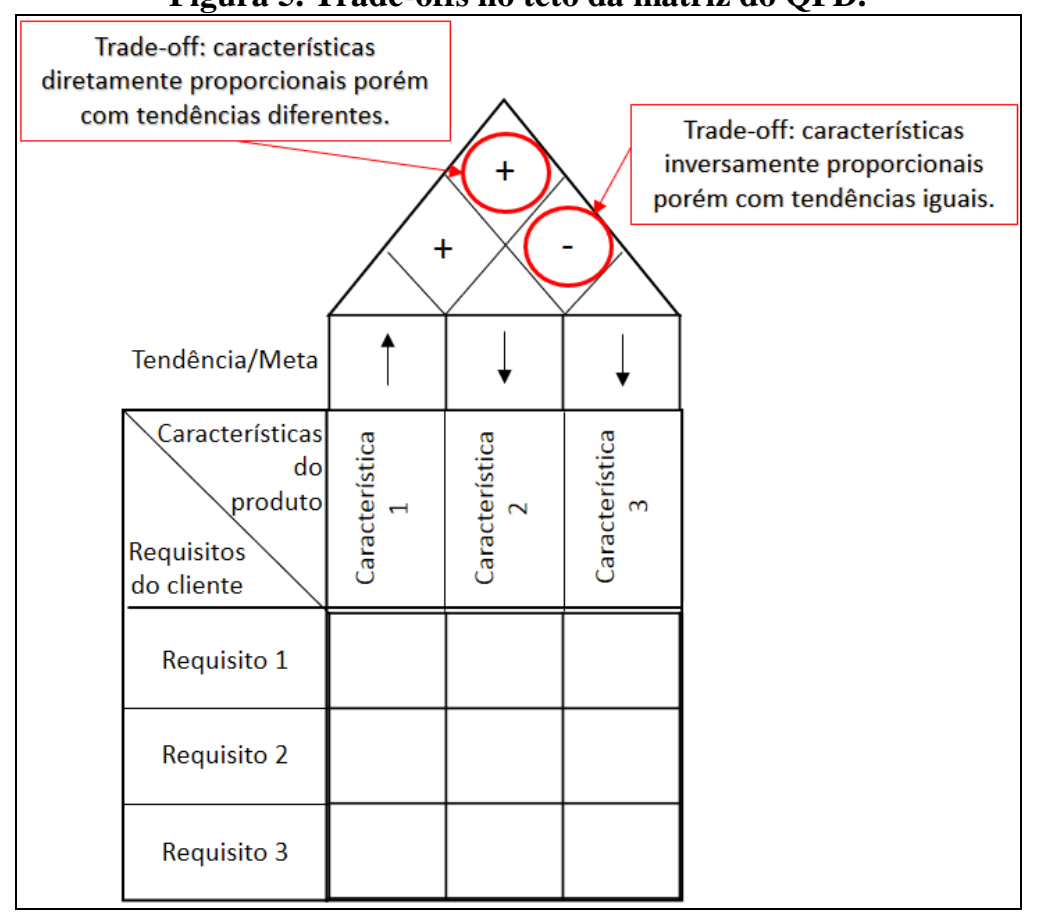

Dessa forma, a casa da qualidade fornece um panorama desses trade-offs relacionados às especificações técnicas do produto enquanto a matriz de característica do subsistema fornece um panorama dos trade-offs das especificações do subsistema. É possível também, utilizando a estrutura de matrizes do QFD, obter um panorama não apenas dentro do subsistema, mas também entre os subsistemas, conforme é apresentado na Figura 6. Estes trade-offs entre subsistemas serão importantes para a integração entre os subsistemas durante o processo de afunilamento no SBCE. O panorama dos trade-offs fornece então um guia para que os tradeoffs de projeto sejam estudados e as curvas de trade-off sejam desenhadas. Essas curvas são utilizadas no processo de afunilamento das alternativas através da sua comparação com os requisitos do cliente e com as demais soluções dos outros subsistemas. 
Figura 6. Panorama dos trade-offs de projeto.

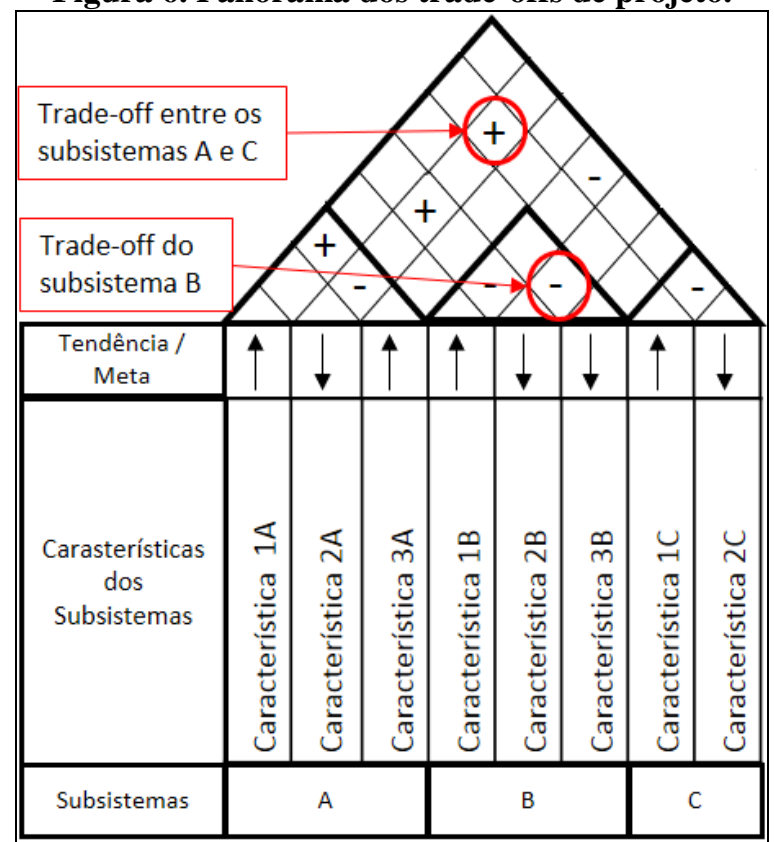

\section{O DESENVOLVIMENTO DO REFRIGERADOR MAGNÉTICO COMPACTO}

O QFD foi utilizado durante o desenvolvimento de um refrigerador inovador cuja tecnologia de refrigeração baseia-se no efeito magnetocalórico que apresentam alguns materiais magnéticos. Esses materiais sofrem uma mudança na sua temperatura quando submetidos a uma variação de campo magnético. O refrigerador magnético foi divido em três subsistemas: regenerador e circuito magnético, sistema hidráulico e de controle e, gabinete e trocadores de calor. Inicialmente, a casa a qualidade do refrigerador magnético foi construída e seu desdobramento para cada um dos três subsistemas foi feito, conforme é apresentado na Figura 7 , que é apresentada miniaturizada devido à confidencialidade do projeto.

Figura 7. QFD do refrigerador magnético compacto.

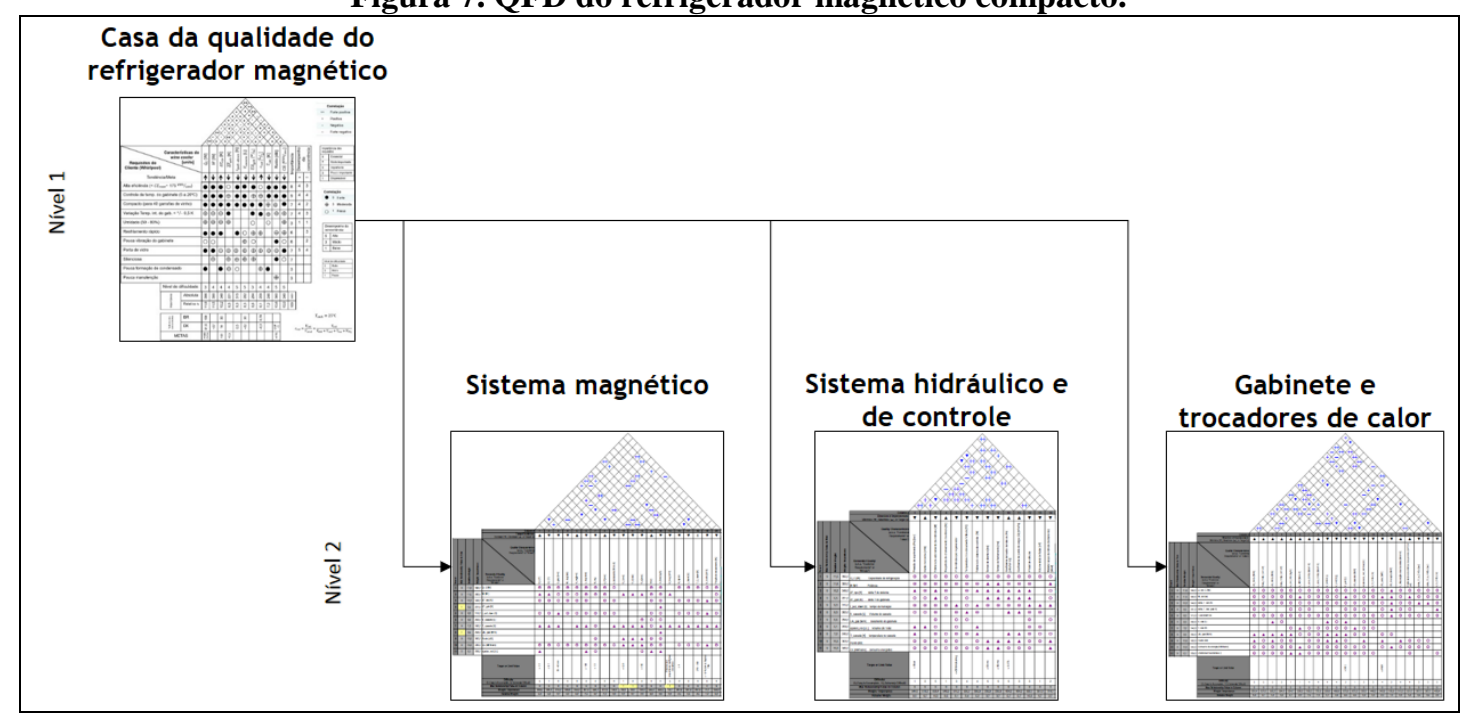


A seguir, uma análise do teto das matrizes foi realizada para encontrar os trade-offs de projeto. Para tanto, além dos tetos das matrizes, construiu-se o panorama dos trade-offs mostrado na Figura 6 e como resultado obteve-se o panorama da Figura 8, que é apresentada de forma miniaturizada devido à confidencialidade do projeto.

Figura 8. Panorama dos trade-offs do refrigerador magnético compacto.

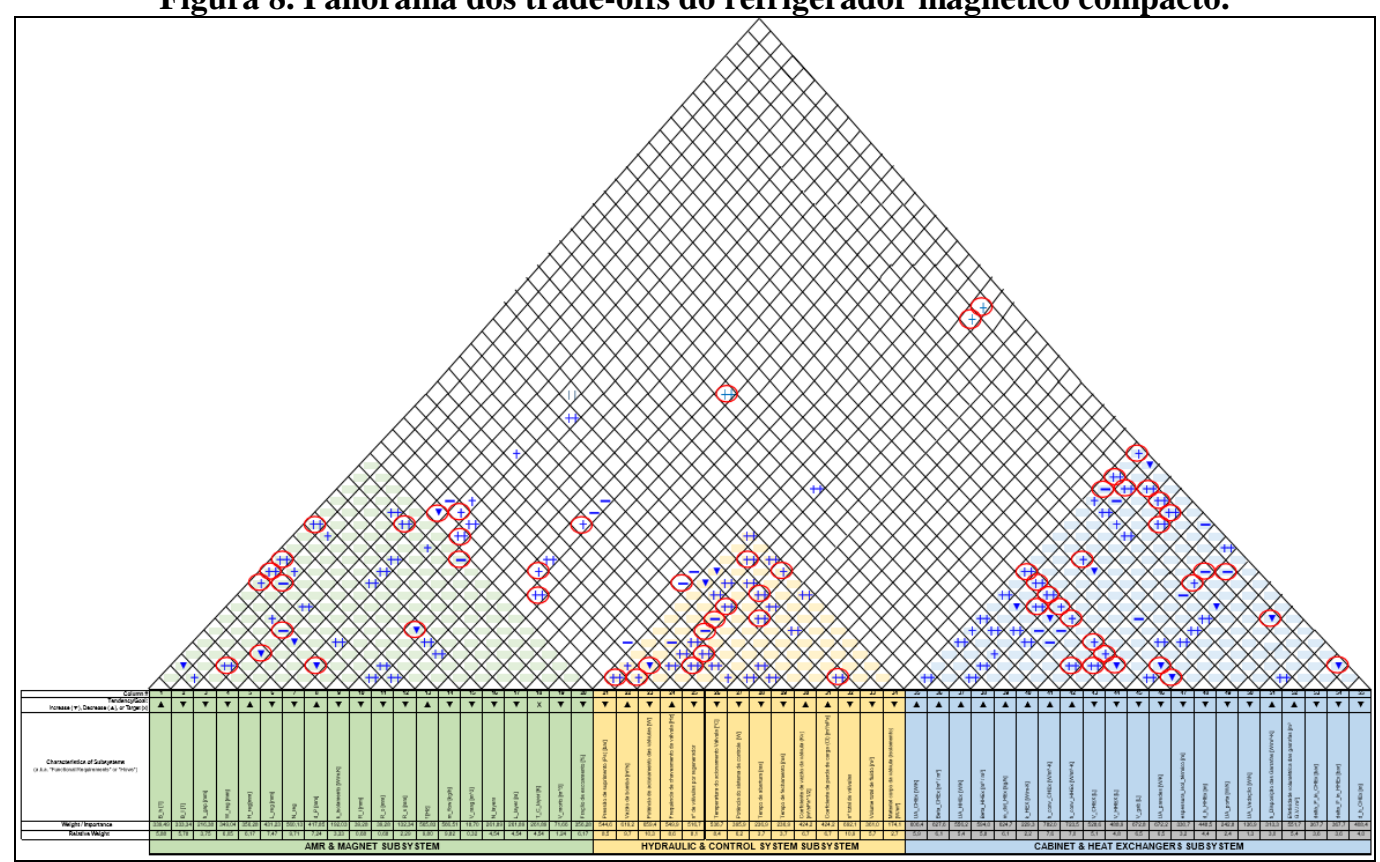

Ao final, e como resultado da análise, o projeto possuiu ao todo 93 trade-offs, distribuídos conforme mostra o diagrama de Venn da Figura 9.

Figura 9. Trade-offs do projeto do refrigerador magnético compacto.

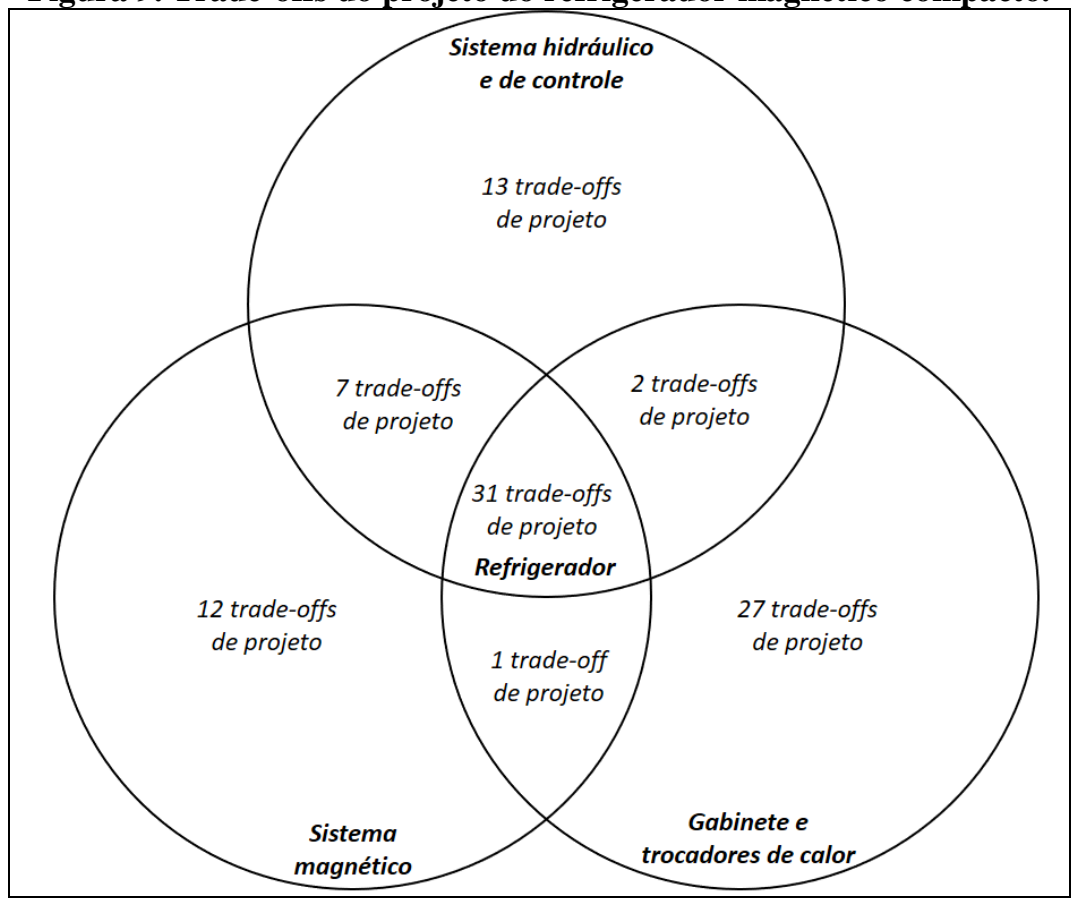




\section{RESULTADOS OBTIDOS}

Através da aplicação do QFD, foi possível desdobrar os requisitos do cliente do produto para os subsistemas. Isso permitiu que os desenvolvedores obtivessem um entendimento uniforme sobre os objetivos do projeto do refrigerador. Observou-se que o QFD promoveu um alinhamento da equipe, o que permitiu a integração dos subsistemas durante o desenvolvimento, principalmente, no processo de afunilamento das alternativas para as características de engenharia do refrigerador. O QFD foi utilizado como referência para a restrição do espaço de projeto, à medida que se definiram as faixas de valores para cada característica de engenharia e essas faixas foram se estreitando. Além disso, com a construção do panorama dos trade-offs de projeto, através das correlações obtidas nas matrizes do QFD, foi possível realizar um plano de estudos desses trade-offs para o afunilamento do espaço de projeto.

\section{CONCLUSÕES}

Foi demonstrado neste trabalho que o QFD é uma ferramenta eficaz para o desdobramento do valor para os subsistemas e para o entendimento e alinhamento dos desenvolvedores para a SBCE. Foi possível também comprovar a contribuição do QFD para o projeto do desenvolvimento de um refrigerador magnético compacto. Além disso, o uso do QFD para a construção de um panorama dos trade-offs de projeto foi importante para nortear os estudos e geração das curvas de trade-off que servem diretamente de insumo para o afunilamento do espaço de projeto. Considera-se que o uso do QFD permitiu a delimitação do espaço de projeto, a comparação desse espaço entre os subsistemas para buscar as intersecções e consequentemente, descartar as regiões do espaço de projeto que não se mostraram viáveis ou compatíveis com os demais subsistemas.

\section{REFERÊNCIAS}

ARACI, Z.C.; AL-ASHAAB, A.; MAKSIMOVIC, M. Knowledge creation and visualization by using trade-off curves to enable set-based concurrent engineering. The Electronic Journal of Knowledge Management, v14, n.1, p.75-88, 2016.

CHENG, L.C. A guide for QFD implementation in product development. Product: Management \& Development, v1, n. 3, p.5-15, 2003.

DIKMEN, I.; BIRGONUL, M.T.; KIZILTAS, S. Strategic use of quality function deployment (QFD) in the construction industry. Building and environment, v40, p.245-255, 2005.

FORD, D.N.; SOBEK II, D.K. Adapting real options to new product development by modeling the Second Toyota Paradox. IEEE Transactions on Engineering Management, v52, n.2, p.175-185, 2005.

FRANCESCHINI, F. Advanced quality function deployment. Florida: CRC Press, 2001. 187 p.

KHAN, M.S.; AL-ASHAAB, A.; SHEHAB, E.; HAQUE, B.; EWERS, P.; SORLI, M.; SOPELANA, A. Towards lean product and process development. International Journal of Computer Integrated Manufacturing, v26, n.12, p.1105-1116, 2013. 
SOBEK II, D.K.; WARD, A.C.; LIKER, J.K. Toyota's principles of set-based concurrent engineering. Sloan Management Review, v40, n.2, p.67-84, 1999.

MARITAN, D. Practical manual of quality function deployment. Suíça: Springer, 2015. 190 p.

MCKENNEY, T.A.; KEMINK, L.F.; SINGER, D.J. Adapting to changes in design requirements using set-based design. Naval Engineers Journal, v123, n.3, p.67-77, 2011.

NATEE, S.; LOW, S.P.; TEO, E.A.L. Quality function deployment for buildable and sustainable construction. Singapura: Singer, 2016. 248 p.

RAUDBERGET, D. Practical applications of set-based concurrent engineering in industry. Journal of Mechanical Engineering, v56, n.11, p.685-695, 2010.

WARD, A.C. Sistema lean de desenvolvimento de produtos e processos. São Paulo: Leopardo editora, 2011. 209 p. 